\title{
Reprocessing of streamer Búzios ANP data: a case study
}

\author{
Marília da Silva Ramos Carneiro, Paulo Crampes, Bruna Carbonesi, Marco Cetale, UFF/DOT/GISIS
}

\section{Copyright 2021, SBGf - Sociedade Brasileira de Geofísica}

This paper was prepared for presentation during the $17^{\text {th }}$ International Congress of the Brazilian Geophysical Society held in Rio de Janeiro, Brazil, 16-19 August 2021.

Contents of this paper were reviewed by the Technical Committee of the $17^{\text {th }}$ International Congress of the Brazilian Geophysical Society and do not necessarily represent any position of the SBGf, its officers or members. Electronic reproduction or storage of any part of this paper for commercial purposes without the written consent of the Brazilian Geophysical Society is prohibited.

\section{Abstract}

We present a case study of 3D streamer Búzios survey, the current most productive field in pre-salt of Brazil, using more recent processing technologies compared to the previous one, in 2012. The purpose is to demonstrate a standard streamer time-processing flow, illustrating what is expected from each step, including basic descriptions, until the Kirchhoff pre-stack depth migration. Preliminary results already shown improvements, compared to the legacy image. Students and non-experts may benefit from this work.

\section{Introduction}

3D towed streamer acquisition and processing has been successfully and widely used since the last decade. Advances in seismic imaging technologies enabled several economical discoveries, including the giants pre-salt fields in offshore Santos basin Brazil. As complex and deeper are the targets, demands to perform new acquisitions or reprocess streamer data has been requested to meet the challenges for exploration, reservoir characterization, and production development.

Seismic acquisition went through outstanding technology development, as well. Due to limitations of streamers, the ocean bottom nodes (OBN) brought the feasibility to have longer offsets, wider azimuths, and higher quality in bandwidth. Despite OBN's advantages, its acquisition is still quite more complex and expensive to be implemented. Therefore, as OBN is not attainable, demands to reprocess streamer datasets have been accomplished using latest processing technologies.

The improvements expected from the reprocessing includes the increase of seismic bandwidth and better subsurface illumination. 3D de-ghosting (Amundsen, 1993, Zhou et. al.,2012), Surface Related Multiple Elimination (SRME), proposed by Verschuur (1992), Full waveform inversion (FWI), as introduced by Tarantola, (1984) and J. Virieux (2009), and Least-squares migration (LSM), presented by Schuster (1993), are novelties developed to meet these demands. The computational power capacity improvement unlocked the use of these tools. Consequently, service provider companies have been hired by the block operator to reprocess the streamer data to include latest technologies.
The offshore Brazil contains several public streamers data available over economically relevant fields. In this vein, we see an opportunity to share, with students and nonexperts, a practical overview of the standard timeprocessing flow. We demonstrate what is expected qualitatively at each processing step, highlighting recent advances.

To place the presenting results in the seismic processing context, let us say that it can be divided into four parts: time-processing, velocity model building (VMB), a.k.a depth-processing, the final migration, and post-processing. As time-processing is ongoing, depth-processing can occur in parallel. Its data is used within the VMB to obtain useful information for the inversion updates (Jones, 2010). The nominated final seismic migration requires the final time-processing data and the final VMB. The postprocessing includes applications of residual denoise, moveout adjustments and amplitude attenuation compensation.

In this work we perform a time-processing flow, using recent technologies, until a Kirchhoff Pre-Stack Depth Migration (KPSDM). Thus, we will be able to compare with the legacy image, a stacked KPSDM volume, processed in 2012. The case study is the 3D streamer Búzios, in Santos Basin, the current largest ultra-deepwater pre-salt oil field in Brazil. The raw data, the legacy image, and the VMB were provided by ANP (Agência Nacional de Petróleo, 1997).

\section{Methodology}

The purpose of time-processing is to prepare data prior input to migration, by solving the following requirement:

- define useful bandwidth,

- remove spurious events (multiples, ghosts),

- correct signal phase and spectrum,

- $\quad$ improve the signal to noise ratio.

For this exercise, we used three sequences, as depicted in Figure 1.

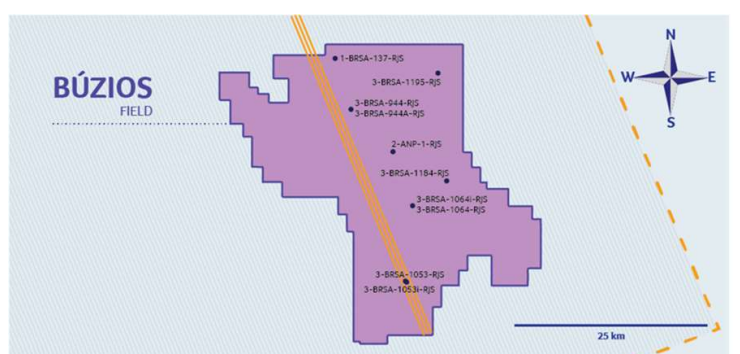

Figure 1 - Búzios field polygon and wells with the processed sequences locations over well 3-BRSA-1053RJS. 
To achieve this, there are usual steps that needs different transform domains and inversions. Figure 2 shows a standard streamer time-processing flow. The order of steps is not commutative and would impacts the results, but it can be varied accordingly to peculiarities and objectives of projects and/or companies. For a comprehensive review of seismic processing, we recommend Yilmaz (2001) and Dondurur (2018).

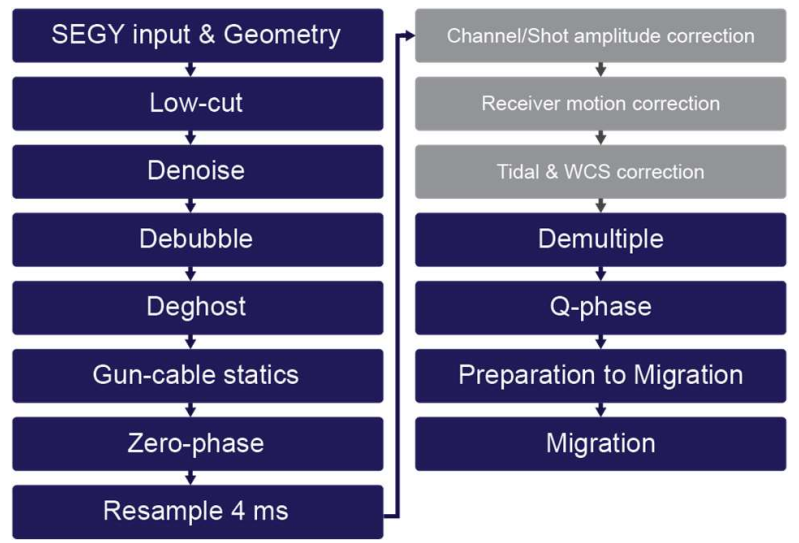

Figure 2 - Streamer time-processing flow. The blue box represents the steps covered in this work.

\section{Examples}

\section{SEG-Y Input \& Geometry}

In this step we convert the recorded (raw) data format into the internal processing software. SEG-Y is one of standard format developed by the Society of Exploration Geophysics to store seismic data (Hagelund \& Levin, 2017). The procedure is made to correlate the trace attribute headers with their bytes to be read. The EBCDIC is a text containing acquisition details and data history. In addition, there are the acquisition report, with general pre-plot info, such as summarized in Table 1 and, for each sequence, exists a log report, informing specific other relevant details, such as shooting sense, inform issues, date, boat speed, etc.

The geometry is made to relate the data real world location with its reference system, called as Datum. This procedure includes define $X Y$ origin, or three points, shooting direction azimuth, and grid space. At this stage, quality control (QC), such as maps of shot, receivers, and fold, must be performed to verify the consistency of the expected geometry information.

\section{Low-cut filter}

Depending on the environment conditions and acquisition instruments, considerable low frequency noise may be present in raw record seismic data. This noise values usually occur below 3 to $6 \mathrm{~Hz}$ and hides part of the data of interest. Its suppression is the first signal processing step and can be made by applying filter in frequency domain (FX), such as Butterworth, trapezoidal, and bandpass (Ross, 2005).

The first recommend analysis is to inspect the seismic data in time-offset domain (TX). Figure 3 shows shot gathers before and after the low-cut filter, with their difference. Also is recommended to sort data in channel/shots (near), as shown in Figure 4, before and after low-cut filter, where is easy to identify some relevant events, such as direct arrival
(DA), water bottom (WB), top of salt (TOS), and first bounce of surface related multiple ( $\left.1^{\text {st }} \mathrm{SRM}\right)$.

A relevant $\mathrm{QC}$ on this stage is to inspect the amplitude spectra. The above left side of Figure 5 shows a much higher amplitude in the lower bands, compared to the other frequencies, while after the low-cut step, the spectrum is more balanced. Cross-check the results by analyzing at the data through the Fourier transform over time and space (FK domain, being $\mathrm{F}$ for frequency and $\mathrm{K}$ the wavenumber) is also another possible QC. At Figure 6 is the FK display before low-cut filter, where is possible to spot a high energy in red, indicated by arrow, and in the right, data after lowcut processing, this energy is vanished.

Table 1 - Acquisition details.

\begin{tabular}{|c|c|c|c|}
\hline \multicolumn{4}{|l|}{ ACQUISITION DETAILS } \\
\hline \multicolumn{4}{|c|}{ Reference system (datum) UTM z $23 \mathrm{~S} 4$} \\
\hline Total area $\left(\mathrm{km}^{2}\right)$ & \multicolumn{3}{|c|}{$408 \mathrm{~km}^{2} / 1428 \mathrm{~km}^{2}$} \\
\hline Line acquisition azimuth & \multicolumn{3}{|c|}{158 degrees } \\
\hline Field low-cut & \multicolumn{3}{|c|}{$4.6 \mathrm{~Hz} / 6 \mathrm{~dB}$ oct } \\
\hline Acquisition year & 2011 & Cable distance $(\mathrm{m})$ & 50 \\
\hline Sample interval (ms) & 2 & Number of channels & 648 \\
\hline Acquisition time (ms) & 9072 & Channel interval (m) & 12,5 \\
\hline Source array amount & 2 & Cable depth (m) & 9 \\
\hline Shot Interval (m) & 25 & First nominal offset $(\mathrm{m})$ & 150 \\
\hline Source depth & 7 & Cable lenght (m) & 8100 \\
\hline Number of cables & 14 & FOLD & 81 \\
\hline
\end{tabular}

\section{Denoise}

The denoise stage consists in attenuate noise and/or edit outlier traces. In other to efficiently separate signal and noise is important to identify their features, such as their shape pattern, frequency content, and origin (Volodya Hlebnikov, 2021). There are two widely common noise types in streamer data: swell noise and seismic interference $(\mathrm{SI})$. In this work swell noise attenuation is performed.

The swell noise occurs due to the tow and disturbance on the streamer motions. Its pattern in the seismic data is a random noise with high amplitude and low frequency, about up to $15 \mathrm{~Hz}$, that increases along the trace time. Other noises, such as SI, can be revealed and better tackled after this step (Volodya Hlebnikov, 2021).

There are several ways to attenuate swell noise. The simplest solution, a low-cut filter, may cause loss of primary signals and resolution. More sophisticated methods may be required. The present work used a band-limited noise suppression for specified smoothed window envelope for noise and signal traces as a complex-trace attribute. For more details, see Oppenheim (1975) and Taner (1979). Figure 7 are displayed shots (a) with swell noise, in (b) data after its attenuation, and (c) their difference. Observe that, at the difference, is not seen any primary leakage. For more examples, we recommend Smith (1999), Volodya Hlebnikov (2021), and Dondurur (2018). 


\section{Debubble}

Bubble effect occurs in the acquisition during the shot of the air-guns causing low frequency reverberations in the data. One air-gun does not produce good signal-noise ratio, because its response generates bubble pulses. In order to make the source signature augmented, modern seismic acquisition fleet contains an array of air-guns with variable volumes, which trigger different energy, designed to overlap the bubble pulses. However, the residual bubble remains in the data and this issue must be addressed.

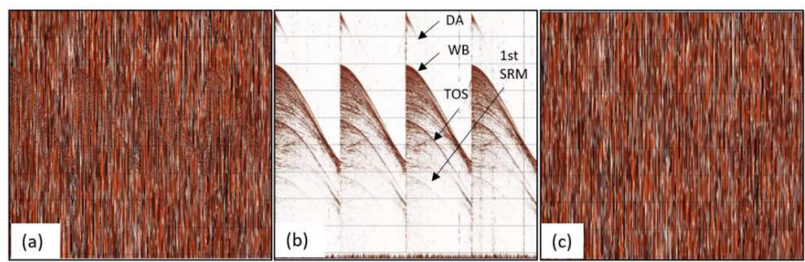

Figure 3 - Shot gathers (horizontal axis is channel and vertical is time): (a) before low-cut filter, (b) after and (c) difference.

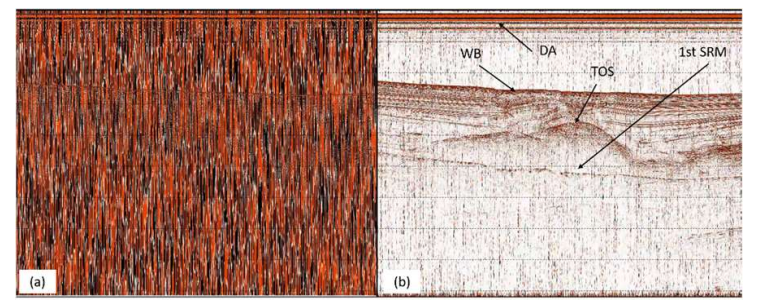

Figure 4 - Low cut in a near channel gather (horizontal axis is shot and vertical is time). (a) without low-cut filter and (b) with low-cut filter with some important events highlighted.

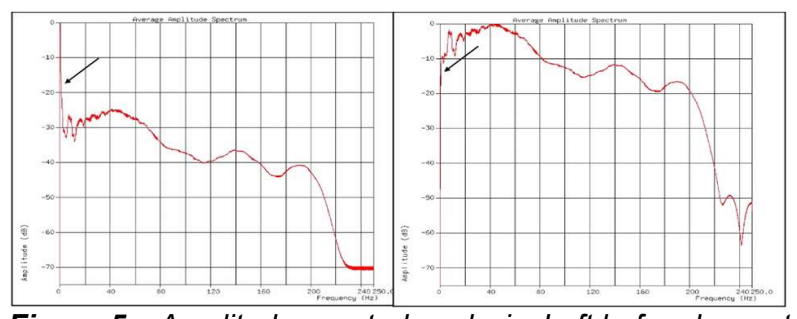

Figure 5 - Amplitude spectral analysis: Left before low-cut filter and right, after. The horizontal axis is frequency for $2 m s$ sample rate data and the vertical the amplitude in $d B$ not normalized.

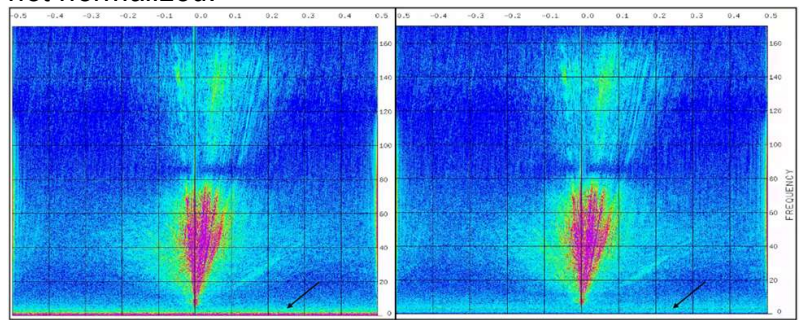

Figure 6 -FK spectral analysis: Left before low-cut filter and right, after. Black arrows indicate the main differences in the low-frequency bands.

A common and simple de-bubble flow consists in deconvolve the source wavelet, statistically derived with near traces. The desired outcome is a trace with representative WB and bubble shapes. This procedure can be performed by flattening the WB and stack a variety of traces to cancel out any geology information. The debubble filter can be extracted with a matching filter between the source signature and the wavelet with the bubble removed. For this, one can design an inversion filter by the Wiener-filter approach (Colin Sargent, 2011). The Figure 8 shows the traces with and without bubble shape, and their matching filter.

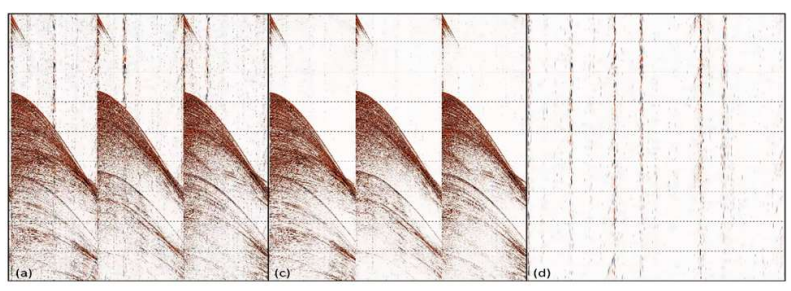

Figure 7 - The effect of mild swell noise attenuation in shot gathers. (a) input, (b) swell-noise attenuated, and (c) the difference.

Figure 9 shows the bubble characteristic in seismic data sorted in channel/shot. Above is data with bubble and below after bubble attenuation. A recommended classic evaluation in the step is check the amplitude spectra, as in Figure 10 , where a peak circled around $10 \mathrm{~Hz}$, that is faded after de-bubble.

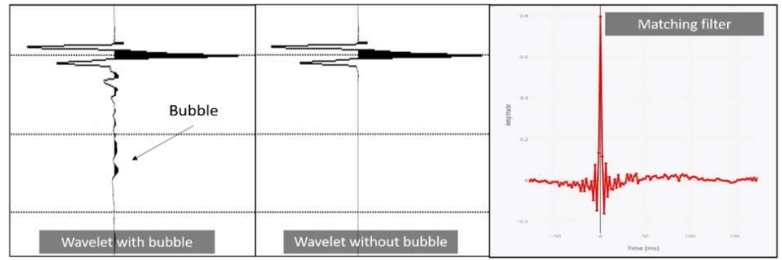

Figure 8 - Wavelet extracted: with and without bubble and the resulted debubble matching filter.

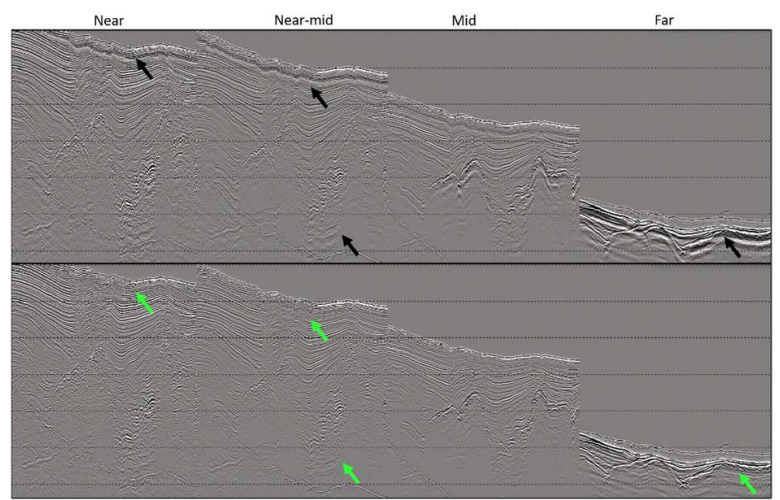

Figure 9 - De-bubble attenuation before and after sorted in channel/shot for near, mid and far channels. Arrows in black indicates the bubble characteristics, and the green's the attenuated result.

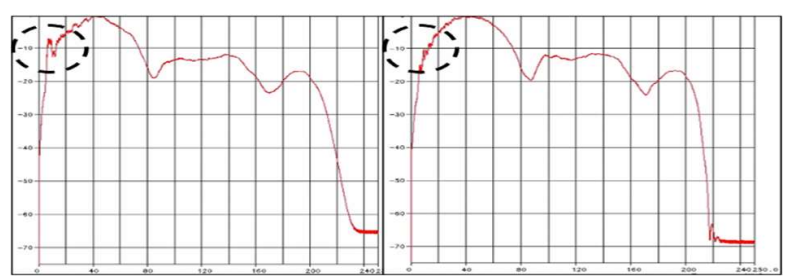

Figure 10 - The amplitude spectra for a channel before (left) and after (right) debubble. Vertical axis is amplitude in $d B$ and horizontal axis the frequency for $2 m$ s sample rate data. 


\section{De-ghost}

Ghost are resulted from the destructive interference of spurious reflection reverberated from the sea surface that superimposes the primary reflections. Figure 11 illustrates that exists three other ray paths beyond the primary reflection (in green). The path in red is the source ghost, blue the receiver's, and orange the source-receiver ghost. At the right side of this figure are the reflections representing the correspondent color for each time-delay path. These time-delays are relies on the source and receiver depths, and would have its polarity reversed as they reflect in the sea surface. They can be represented by

$$
\Delta t=\frac{2 d \cos \theta}{v}
$$

where $\Delta t$ is the ghost delay, $d$ are the source or receiver depths, $\theta$ the path incidence angle, and $v$ the water velocity. Theses extra reflections causes destructive interference at specific frequencies, a.k.a, notches, causing resolution limitations. The so-called frequency notch $f$ can be computed by

$$
f=\frac{v}{2 d} \cos \theta \text {. }
$$

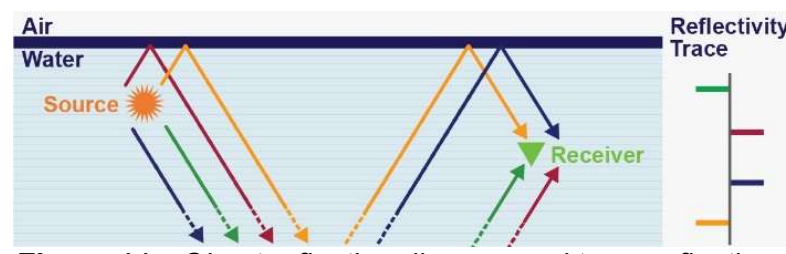

Figure 11 - Ghost reflection diagram and trace reflections. Green path represents the primary's, red is the source ghost, blue is the receiver ghost, and yellow the sourcereceiver ghost.

The goal of the de-ghost is to obtain a broader bandwidth, by having the frequency notches removed, which makes the amplitude spectra flatter. This step is one of the main advances, since Búzios legacy data was first processed in 2012. To obtain the ghost free wavefield the usual procedure is using an inverse filter considering the ray parameter $(\tau-p)$, in order to cover delays as the incidence angle increases.

In the streamer Búzios reprocessing the ghost attenuation achieved a satisfactory result. Figure 12(c), in the wiggle view, indicating that the water bottom is in minimum phase with negative peak. The right side are the amplitude spectra, where the black arrow indicates a notch around $80 \mathrm{~Hz}$ before and after deghost. Note that the data has its frequency content broader and more balanced (flatter). If we go back to the FK display, shown in Figure 5 and 6, it was already possible to see the notch around the frequency $80 \mathrm{~Hz}$.

Notice that deghost process boosts amplitude, therefore, if prior stages are not made properly, noise is boosted, as well. In this case, one can see, in the Figure 12(d), at the low frequencies, some peaks indicating residual bubbles uplifted, that is recommended to be addressed.

As can be observed in Figure 12 (b) and (d), the Nyquist is $125 \mathrm{~Hz}$, indicating that data is sampled by $4 \mathrm{~ms}$. Although the resample being recommended to be applied after guncable statics and zero-phase steps, to have better precision and preservation of frequency content, the de- ghost procedure is computationally expensive. Hence, with the purpose to save time, we made the resample prior performing it.

\section{Gun-cable statics}

In the acquisition, source and receiver are placed below sea level and at different depths. This difference should be removed, due to migration algorithm requirements. The gun-cable static is aimed to move the shots to the sea level, and the way to achieve it, is just by remove a constant value $c$. This value is obtained simply by computing the following equation, giving the depths of source and receiver $\left(s_{z}\right.$ and $\left.r_{z}\right)$ and the water velocity $v$ :

$$
c=\frac{s_{z}+r_{z}}{v}
$$

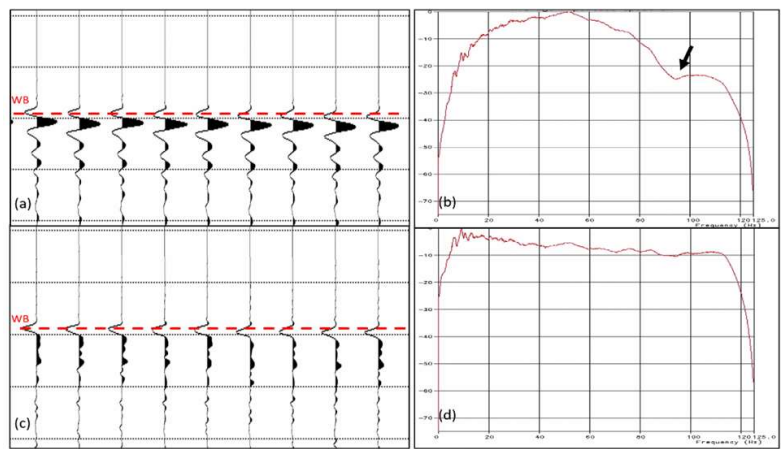

Figure 12 - Two displays before (above) and after deghost (below). Water bottom displayed in wiggle view are shown in (a) and (c) and the respective amplitude spectra in (b) and (d) for $4 m s$ sample rate data.

In streamer Búzios case the static value removed is $10 \mathrm{~ms}$

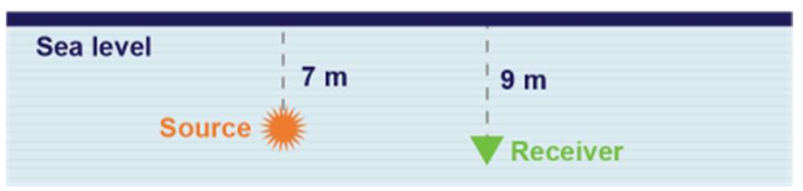

Figure 13 - Illustration of source and receiver depths below sea level.

\section{Zero-phase filter}

For being an explosive source of energy, the seismic data comes from the acquisition with minimum phase, in which the peak is with less delay and concentrated as near as possible of the shooting start time. The zero-phase step is a convention in which the reflectors (interface between layers) is a peak. The zero-phase filter should make the trace function shape approximately symmetric.

In practical view, as can be seen in Figure 14, a common methodology is extract the wavelet, statistically from the near traces, with the aim to design a filter before applying it to all shots. The zero-phase operator is obtained by Fourier transforms, by shifting component frequencies (Dondurur, 2018 and Yilmaz, 2001).

The Figure 15 shows the WB peak before and after zerophase filter application. Above can be observed in wiggle view data in minimum phase (a) and in zero-phase (b). Observe that the WB waveform are more symmetric. The bottom images in Figure 15 shows the zero-phase filter effect in a near channel, where the WB peak became positive (black), with symmetric white side lobes. 


\section{Resample $4 m s$}

The resample is made to reduce computational cost and memory storage. An anti-alias filter considering the Nyquist frequency $(125 \mathrm{~Hz}$ for $4 \mathrm{~ms})$ is necessary to avoid frequency content wrap-around.

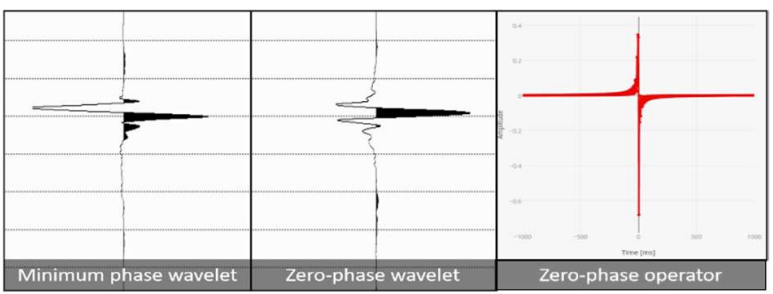

Figure 14 - Zero-phase wavelets: in left with minimum phase, middle with zero-phase filter applied, and the zerophase operator designed.

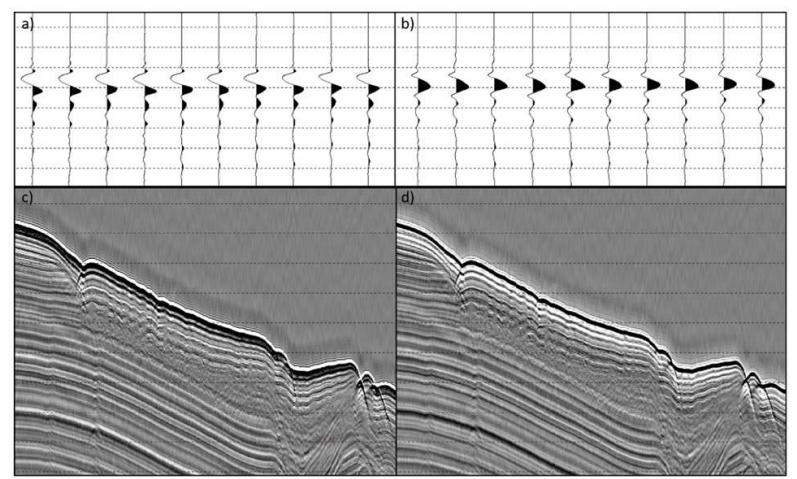

Figure 15 - The zero-phase pattern. Above is a near trace in wiggle view: (a) is before and (b) is after the zero-phase filter. Below illustrates the zero-phase effect in WB shots: (c) before filter and (d) after filter.

\section{De-multiple}

Multiples occurs in marine data due to extra reflections mixed with the primary's ones. They are different of the ghosts, since the spurious reflection comes after reverberating twice, or more times, the subsurface. The first order (bounce) of surface related multiples (SRM) has similar energy as the water bottom and twice its time (and so on for other multiple orders). In the case of Santos basis, for instance, the first bounce hides the pre-salt events, since the sea floor ranges around $2 \mathrm{~km}$. Therefore, SRM is the main multiple type tackled in marine processing.

The Surface Related Multiple Elimination, known as SRME (Verschuur, Seismic multiple removal techniques, 2006), and Radon are the usual methodologies to attenuate multiples. One of the main benefits of SRME is attenuate the near trace multiples, not covered by the previous methodology, Radon domain de-multiple, which yet, is still recommended, but as a complementary step.

The SRME method uses data to predict the sea surface related multiples, by convolutional process of sourcereceiver traces. Its procedure is separated in two parts: first multiple estimation and then an adaptive subtraction is made without damage the hidden primaries. Figure 16 shows input shots, SRME model, and the adaptive subtraction result.

In the Radon methodology the multiples can be differentiated in TX domain if NMO is applied, where primaries are expected to be flat and multiples with moveouts. In the Radon domain is easier separate flat events, from others since they are located at approximately $p=0$. However, it works only for mid-far offsets. In the Figure 17 we show a CDP/Offset gather illustrating a Radon de-multiple tackling the SRM, as well.

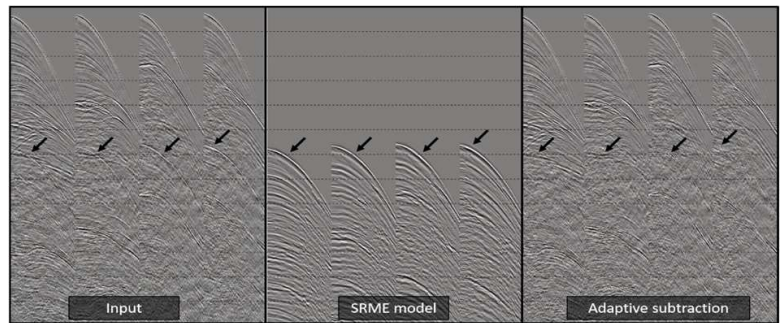

Figure 16 - SRME shots: left before SRME, in the middle the SRME model, and in the right the adaptive subtraction result. Black arrows indicate the first order of SRM.

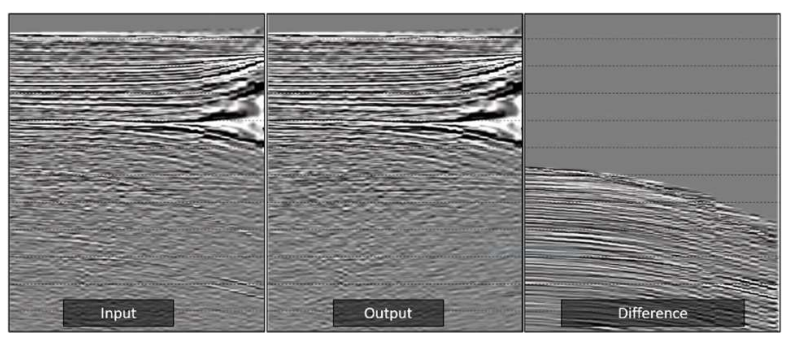

Figure 17 - Radon de-multiple. Left in the input, in the middle the output, and in the right the difference with gain.

\section{Q-phase (dispersion correction)}

Absorption effects occurs in trace amplitude and phase while the wavefield propagates in the Earth's subsurface, as the traveltime and frequency increases. The inverse of $Q$ is a dimensionless quantity to compensate dispersion and dissipation. The purpose is enhance seismic vertical resolution, correcting timing and lithological identification.

The Q-phase only is recommended to be applied prior the migration (in time-processing), in order to avoid some high frequency noise gain energy. After the migration, a stable, then Q-amplitude should be applied, making the amplitude spectra of higher frequencies more flatten. For more details we recommend Wang (2004).

The Q-phase effect can be seen in the seismic data on Figure 18. The result is expected to be subtle, giving the visual impression that occurs a time variant shift going up, and should not affect the amplitude spectra.

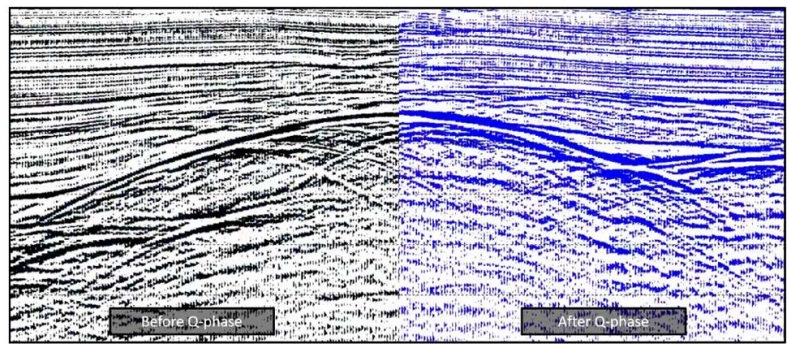

Figure 18 - Q-phase application effect in a mid-channel: black is before, blue is after. 


\section{Data preparation for migration}

The goal of this step is to correct acquisition irregularities that could cause migration smiles and unbalancing the amplitude. Each migration algorithm type should have their respective preparation.

With the objective to compare our reprocessing with the legacy image, here we prepared data for Kirchhoff migration. In this case, the step is called "regularization". The regularization is aimed to transform traces, with defined grid and offset panels, to be perfectly placed in the bin center.

For this work, instead of performing full regularization, we only regularized to offset values, in order to have regular offset volume planes. The goal is to consider these new offset values in the bin center, with rounded value and fixed increment. This is made by gathering similar offsets and applying a partial NMO. The fold resulted in 81 . The traces remain the same and no binning (trace discard, remaining only those as close as possible to the bin center) was made.

\section{Migration}

The migration is the imaging process, which is aimed to properly correct the subsurface reflection events to their true locations (Yilmaz, 2001). This process requires, beside the time-processed data, an accurate velocity model of the subsurface.

With the view to perform a comparison with the legacy migration, as fair as possible, we performed a Kirchhoff pre-stack depth migration (Schneider W. , 1978), using the provided legacy's isotropic interval velocity model in depth. As in the legacy volume, the grid space is 12.5 meters, in crossline direction, and $25 \mathrm{~m}$ in inline. The migration output has sample rate of 4 meters, aperture of $4000 \mathrm{~m}$. Figure 19 shows improvements delivered by the reprocessed data at the sediment region, when compared with the legacy KPSDM volume stack.

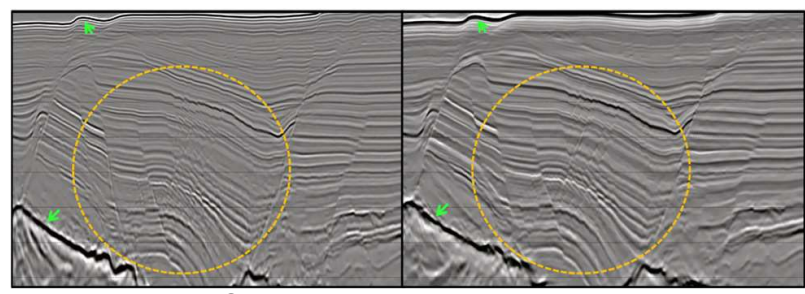

Figure 19-KPSDM stacked at the sediment region (Inline 4118) comparison: legacy (left) and the reprocessed (right). It is possible to observe that ghost side-lobes were attenuated (green arrows) and the center fault region is better imaged (orange dashed circle).

\section{Conclusions}

We have performed a full streamer time-processing workflow, from raw seismic data to migrated data. Key time-processing step results were highlighted and displayed, in order to illustrate their result effects. In addition, more recent processing technologies were applied to be compared after migration and stack to the previous processed image.

We emphasize that this is a part of a research project, with goals to develop algorithms to real applications. For a complete time-processing, one should encompass some processes not covered on this work, such as other denoise approaches, channel amplitude correction, receiver motion correction, water column statics, and full regularization prior migration. Also, one might use more sequences to have more migration aperture, getting the best possible image in the complex and deeper regions.

\section{Acknowledgments}

We would like to thank PETROBRAS for the financial support. We also acknowledge Jaime Yanez and Emerson-Paradigm for the technical support and the academic license processing software. We thank Rodrigo Stern for his constant quest to improve the computational resources for GISIS group and to UFF's infrastructure.

\section{References}

Agência Nacional de Petróleo, G. N. (1997) http://www.anp.gov.br.

Amundsen, L. (1993). Wavenumber-based filtering of marine point-source data. GEOPHYSICS, 58, 1335-134.

Baysal, E., Kosloff, D., \& Sherwood, J. W. (1983). Reverse Time Migration. Geophysics, 1514-1524.

Colin Sargent, R. W. (2011). Improving the interpretability of airgun seismic reflection data using deterministic filters: A case history from offshore Cape Leeuwin, southwest Australia. Geophysics, 76.

Dondurur, D. (2018). Acquisition and Processing of Marine Seismic Data. Elsevier.

Dondurur, D. (2018). Acquisition and Processing of Marine Seismic Data. Elselvier.

Hagelund, R., \& Levin, S. A. (2017). SEG-Y_r2.0: SEG-Y revision 2.0 Data Exchange format.

J. Virieux, S. O. (2009). An overview of full-waveform inversion in exploration geophysics. Geophysics, WCC1-WCC26.

Jones, I. F. (2010). An Introduction to: Velocity Model Building. EAGE Publications.

Oppenheim, A. V. (1975). Digital Signal Processing

Ross, D. (2005). Ship sources of ambient noise. IEEE Journal of Oceanic, 30, 257-261.

Schneider, W. (1978). Integral formulation for migration in two andthree dimensions. Geophysics, 43, 49-76.

Schneider, W. (1978). Integral formulation for migration in two andthree dimensions. Geophysics, 43, 49-76.

Schuster, G. T. (1993). Least-Squares Cross-Well Migration. 63rd SEG, pp. 110-113.

Smith, J. G. (1999). Amplitude and phase effects of weather noise. SEG .

Taner, M. T. (1979). Complex seismic trace analysis. Geophysics, 44, 1041-1063.

Tarantola, A. (1984). Inversion of seismic reflection data in the acoustic approximation. Geophysics, 1259-1903.

Verschuur, D. J. (1992). Adaptive surface-related multiple elimination. Geophysics, 57, 1166-1177.

Verschuur, D. J. (2006). Seismic multiple removal techniques. EAGE.

Volodya Hlebnikov, T. E. J. (2021). Noise types and their attenuation in towed marine seismic: A tutorial. Geophysics.

Wang, Y. (2004). Q analysis on reflection seismic data. Geophysical Research Lettes.

Yilmaz, Ö. (2001). Seismic Data Analysis. Society of Exploration Geophysicists.

Zhengzheng Zhou, M. C. (2012). Analysis of a Broadband Processing Technology Applicable to Conventional Streamer Data. First Break, 30, 77-82. 